The Impact of Large-Scale Surveys on Pulsating Star Research

ASP Conference Series, Vol. 203, 2000

L. Szabados \& D. W. Kurtz, eds.

\title{
Large-Scale Surveys and the GCVS
}

\author{
N. N. Samus, E. V. Kazarovets \\ Institute of Astronomy, 48 Pyatnitskaya Str., Moscow 109017, Russia \\ O. V. Durlevich \\ Sternberg Astronomical Institute, 13 Universitetsky Ave., Moscow \\ 11989g, Russia
}

\begin{abstract}
We briefly discuss changes in the work on the General Catalogue of Variable Stars (GCVS) necessary in the era of automatic variability discoveries and large-scale surveys.
\end{abstract}

The style of the GCVS work must change in the era of large-scale surveys. The 74th Name-list of variable stars (Kazarovets et al. 1999), specially created for variable stars discovered by the Hipparcos (HIP) mission, is the largestever, containing 3157 variables announced in a single reference! This experience teaches several lessons.

The HIP team initially suggested to us that we give GCVS names to 5665 stars, of which 3157 were then named by us. The majority of the stars not named were included in the NSV Supplement (NSVS, Kazarovets et al. 1998); a small number of stars were found to already have GCVS or NSV identifications. HIP variables included in the NSVS (1956 objects) are mostly stars with information insufficient for determination of their variability types. Of the newly named HIP variables, nearly $50 \%$ (1464 stars) have uncertain classification. We have encountered a number of cases of spurious variability in the HIP data; due to wrong identifications in the input catalogue, some stars were given completely wrong spectral types, resulting in erroneous photometric reductions.

We see two important problems with large-scale surveys for the GCVS: 1) Observations are scheduled for reasons far from those of variable-star research. So it is difficult to derive types for some stars. There are almost no Cepheids among new HIP variables (11 stars for all Cepheid subtypes, with uncertain classification for 10 of them). The HIP team admits that it was especially diffcult to derive periods in the typical Cepheid range from their data. The number of RR. Lyrae stars is also low (28 stars, only 6 of them certain). On the other hand, $\delta$ Sct and SX Phe stars are well represented (97 stars, 68 of them certain). Semiregular and irregular variables give 1692 stars, $54 \%$ of the sample. Thus, the distribution of the stars over types is rather peculiar. 2) Insufficient attention to identifications makes it more difficult to analyse photometric data. In the HIP project, extensive use was made of SIMBAD data base. The latter contains mistakes in identifications (resulting, in particular, from many catalogues having very bad positional accuracy). We have discovered more than 500 mistakes or imperfections in SIMBAD identifications. 
The USNO A1.0/A2.0 catalogue makes it possible to drastically improve the situation with positions of variables. We have launched an extensive project with the aim to present accurate positions for as many old GCVS stars as possible. We have practically finished this work for the stars of the first GCVS volume, whenever possible attempting also to give proper motions. For new GCVS, NSV, and NSVS versions, regularly corrected and improved, see our web site at http://ww.sai.msu.su/groups/cluster/gcvs/gcvs/

where the new co-ordinates for Vol. I will soon appear. Work on identifications and positions of variables is also being done by other researchers. Comparison of results makes it possible to find mistakes and solve complicated cases.

The HIP Name-list was our first experience with large surveys. New special Name-lists will follow. If a survey presents well-structured information on variables, it becomes self-sufficient to a considerable degree, making delays with GCVS naming not so worrying. Almost perfect is the presentation of data in OGLE publications. However, the OGLE classification (eclipsers, short-period pulsators, miscellaneous periodic variables) is insufficient for our traditions. On the other hand, the availability of data from some other surveys (e.g., MACHO) is a serious problem, to be discussed at the IAU level. If thousands of stars are being discovered, with only a small part of them announced in a manner sufficient for identification, the rest of the discoveries are effectively lost.

Important lessons follow also for the GCVS system of classification. It was clear that the system needed improvement even before the HIP mission. The most serious consequences of the HIP findings for the GCVS pulsating-star classification system are the following. 1) Among red stars, we meet probable pulsators not only among supergiants and giants but also among subgiants. For subgiants and especially for dwarfs, it is not easy to decide whether the observed variability is due to rotation or to pulsation. Already, it seems justified to introduce a type for semiregular pulsating red subgiants. 2) Among red giants and supergiants, the HIP team derived quite a number of short periods of variability (of the order of days). If real, such periods would cause problems for interpretation and make it necessary to revise the classification of numerous "old" SRB, SRC, LB, and LC stars for which such a behaviour was even not looked for. 3) Among O-F stars, we meet variables, probably pulsating ones, not quite satisfying GCVS criteria for DSCT, SXPHE, BCEP, or ACYG stars. The $\gamma$ Dor stars, recently suggested as a new type of pulsating variables, are obviously a related phenomenon, as are the Maia variables, suggested long ago and not understood until recent improvements of stellar opacities.

It is tempting to introduce many new types of pulsating variable stars. But the existing version of the classification system already contains too many types to be easily understood by the users. We would be grateful for suggestions aimed at a clear and minimally sufficient system of variable-star classification.

Our GCVS work is supported, in part, by the Russian Foundation for Basic Research (grant 99-02-16333) and by the Federal Program "Astronomy".

\section{References}

Kazarovets, E. V., Samus, N. N., \& Durlevich, O. V. 1998, IBVS, No. 4655

Kazarovets, E. V., Samus, N. N., Durlevich, O. V., et al. 1999, IBVS, No. 4659 